\title{
Fast positronium formation and dissociation at surfaces
}

\author{
D. W. Gidleya) and D. N. McKinsey \\ Physics Department, University of Michigan, Ann Arbor, Michigan 48109 \\ P. W. Zitzewitz \\ Department of Natural Sciences, University of Michigan-Dearborn, Dearborn, Michigan 48128
}

(Received 22 March 1995; accepted for publication 17 April 1995)

\begin{abstract}
The origin of short-lived components in the annihilation lifetime spectrum of positronium (Ps) is shown to be due to fast Ps that is collisionally dissociating at the surfaces of the surrounding confinement cavity. The results are consistent with a model of fast (10-100 eV) Ps production by backscattered positrons from the incident beam. It is found that the typical lifetime of dissociating Ps scales with the mean free path of the cavity, and the relative formation intensity depends inversely on the incident positron beam energy. This ubiquitous effect will be present in any Ps formation experiment involving a free surface and can only be eliminated at beam energies less than $10 \mathrm{eV}$. More practical methods of minimizing the undesirable systematic effects of fast Ps quenching in depth-profiled positron lifetime spectroscopy and in precision Ps decay rate measurements will be discussed. (C) 1995 American Institute of Physics.
\end{abstract}

\section{INTRODUCTION}

In the last 15 years low-energy, focused beams of positrons have been used in condensed-matter physics ${ }^{1}$ and in fundamental tests of quantum electrodynamics. ${ }^{2,3}$ More specifically, the diffusion of thermalized positrons back to the surface and the subsequent capture of an electron to form positronium (Ps) has been used as a depth-profiled probe of metals and semiconductors ${ }^{1}$ and as the mechanism for forming Ps in precision Ps decay rate measurements. ${ }^{2}$ A complicating factor in all of these experiments is Ps that is formed from epithermal and/or backscattered positrons that have not participated in the diffusion process. This effect produces speciously high Ps formation at low implantation energies ${ }^{4}$ and the Ps so formed has higher energy than that formed from thermalized positrons. ${ }^{5}$

This fast Ps has typically been dealt with by eliminating the data acquired below $5 \mathrm{keV}$ beam energy. ${ }^{4}$ For metals and semiconductors that have high positron stopping power and relatively long positron diffusion length, this loss is acceptable. However, positron beams are beginning to be used in polymer studies ${ }^{6,7}$ in which much of the physics occurs below $5 \mathrm{keV}$ beam energy. Furthermore, there is recent interest in applying the well-developed bulk polymer technique of positron lifetime spectroscopy ${ }^{8}$ to depth-profile polymer surfaces, thin films, and interfaces with beams. ${ }^{6,7}$ We will show in this paper that the fast Ps produced from backscattered positrons can readily dissociate on surrounding surfaces to produce fast decaying components in a lifetime spectrum that will need to be accounted for in such polymer studies. Moreover, we can now identify this dissociating Ps as the main systematic impediment to improving the accuracy of precision Ps decay rate measurements. ${ }^{2}$ The goal of this paper is therefore twofold: to improve the understanding of the fast Ps formation mechanism using a variety of target materials and over a wide range of beam energies, and to explore the dissociation probability for this fast Ps when it encoun-

\footnotetext{
a)Electronic mail: Gidley@miphys.physics.Isa.unich.edu
}

ters a surface. Compared with the previous studies of fast Ps formation, ${ }^{4,5}$ we will use the complementary technique of positron lifetime spectroscopy in this work. Not only does this present a systematically different view of the process, but it directly probes the lifetime of dissociating Ps as required for beam-related lifetime studies.

\section{EXPERIMENT}

The positron beam and timing apparatus used are those of the vacuum Ps decay rate experiment described in Ref. 2. The positron beam (Fig. 1) is produced by a ${ }^{22} \mathrm{Na}$ source and a tungsten ribbon (venetian blind) moderator. Approximately $10^{-4}$ of the beta decay positrons are reemitted from the moderator with an energy of about $3 \mathrm{eV}$. These positrons are accelerated and focused electrostatically into a $2 \mathrm{keV}$ beam that strikes a Ni foil remoderator. Approximately $20 \%$ of the positrons incident on the remoderator are reemitted with an energy of $2 \mathrm{eV}$. Secondary electrons simultaneously ejected from the $\mathrm{Ni}$ foil are detected by a channel electron multiplier array, forming a start timing signal. The remoderated positrons are again accelerated and focused by electrostatic lenses and are transported $85 \mathrm{~cm}$ to a cavity where Ps is formed and confined.

The cavity, $3.0 \mathrm{~cm}$ in diameter, $3.3 \mathrm{~cm}$ long, is machined from either aluminum or stainless steel. Positrons enter through the $5 \mathrm{~mm}$ diameter hole and strike the back wall that is separately biased so that the incident energy of the positrons can be varied. The positrons are implanted into the material at a depth dependent on their incident energy. They scatter and lose energy, some return to the surface, capture an electron, and escape into the cavity as positronium. We varied both the incident positron energy and the Ps formation surface, using stainless steel, aluminum, molydbenum, lead, and fumed $\mathrm{MgO}$ powder.

Positronium confined in the cavity annihilates into gamma rays that are detected by scintillators surrounding the cavity. The time between emission of the positrons at the remoderator and detection of the annihilation gamma(s) was 


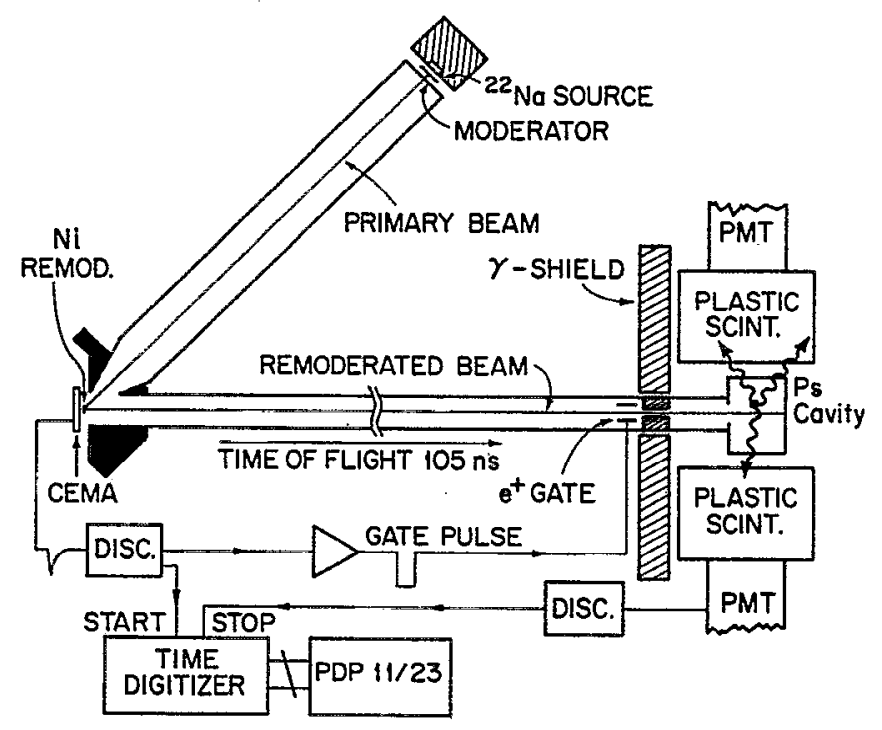

FIG. 1. The remoderated positron beam and timing apparatus used in this experiment.

measured and recorded to form the Ps lifetime spectrum. Triplet, or orthopositronium ( $o-\mathrm{Ps})$, decays with a free-space lifetime of $142 \mathrm{~ns}$, but it can also decay as the result of surface collisions. We were able to fit the $o$-Ps spectrum reliably within $20 \mathrm{~ns}$ after $o$-Ps formation.

\section{RESULTS AND DISCUSSION}

At each incident beam energy the lifetime spectrum was fitted to two exponential components: a long lifetime component (decay rate $\lambda_{T}$ and intensity $I_{T}$ ) that accounts for Ps that survives collisions with the cavity surfaces and a fast lifetime component characterized by a decay rate $\lambda_{f}$ and intensity $I_{f}$. As shown in Fig. 2, this method clearly oversimplifies the fast component since the fitted values of the decay

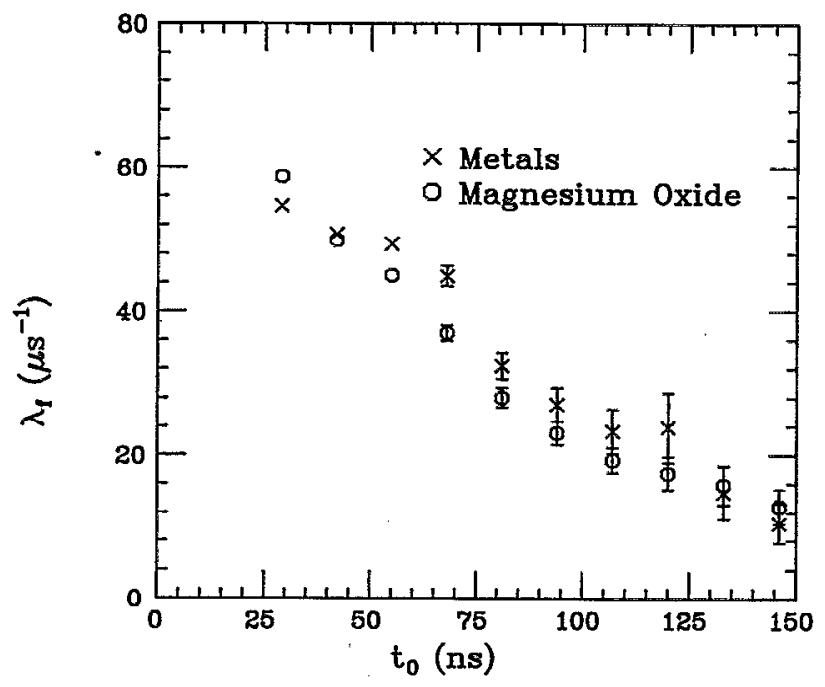

FIG. 2. Fitted decay rate $\lambda_{f}$, of the short-lived Ps component, as a function of start time of the fit, $t_{0}$, for metallic and fumed- $\mathrm{MgO}$ positronium formation surfaces.

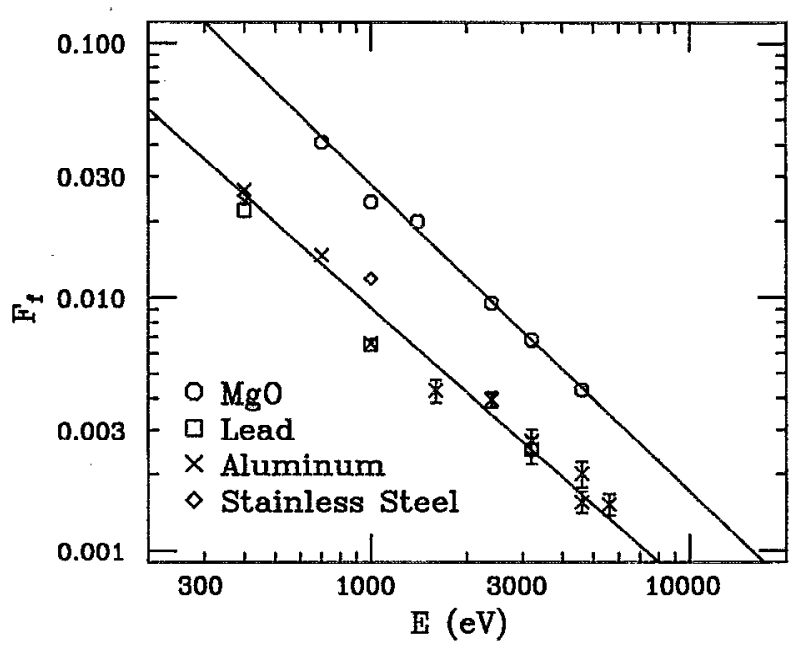

FIG. 3. The fraction $F_{f}$ of all annihilations that occur in the fast Ps component as a function of the positron beam energy $E$.

rate $\lambda_{f}$ and intensity $I_{f}$ decrease as the start time $\left(t_{0}\right)$ of the two-exponential fit is stepped out. For consistent and systematic comparison we determine the fitted intensity at $t_{0}=42$ ns and calculate the fraction of all annihilations occurring in the fast component. This fraction, $F_{f}$, is shown in Fig. 3 as a function of the positron beam implantation energy $E$. Note that $F_{f}$ decreases roughly as $1 / E$ and is about $3 \times$ higher for $\mathrm{MgO}$ formation surfaces than metal surfaces.

If we assume that the fast component is the result of Ps annihilating during wall collisions with some probability per collision, $P_{a}$, then the fitted decay rate can be used to determine the kinetic energy of the Ps. Kinetic theory and our measurements of long-lived $o$-Ps in evacuated cavities ${ }^{2}$ show that the annihilation of $o$-Ps on cavity walls results in a fitted decay rate given by

$$
\lambda_{f}=\lambda_{T}+\bar{v} \frac{P_{a}(\bar{v})}{(4 V / S)},
$$

where $\lambda_{T}$ is the vacuum decay rate, $P_{a}(\bar{v})$ is the probability that Ps with an average velocity of $\bar{v}$ will annihilate in a wall collision, and $4 \mathrm{~V} / \mathrm{S}$ is the mean free path in a cavity of volume $V$ and surface area $S(4 V / S=2 \mathrm{~cm}$ in our cavity). From Fig. 2 we see that at early times (small $t_{0}$ ) $\lambda_{f}$ is approximately $60 \mu \mathrm{s}^{-1}$, a Ps lifetime of only $17 \mathrm{~ns}$. The kinetic energy of Ps decaying this quickly is given by $E_{\mathrm{Ps}}=6.5$ $\mathrm{eV} / P_{a}^{2}$. The lower limit of kinetic energy, corresponding to $P_{a}(\tilde{v})=1$, is thus $6.5 \mathrm{eV}$. Clearly, Ps in this $17 \mathrm{~ns}$ component has relatively high energies compared to Ps formed from thermal positrons, which has kinetic energies typically less than $3 \mathrm{eV}$ for most metals. ${ }^{9}$ Such fast Ps has been observed in Ps velocity spectroscopy ${ }^{5}$ and the amount of fast Ps is observed to decrease rapidly as the positron implantation energy approaches several kilo-electron-volts, similar to the results shown in Fig. 3.

The formation of fast Ps must be due to positrons that are so shallowly implanted in the target that they cannot thermalize before returning to the surface where they capture an electron to form energetic Ps. ${ }^{4,5}$ A detailed understanding 
of the dynamics of this process would require convolution of the nonthermal positron energy spectrum with the energydependent electron-capture cross section. In principle, the target material and surface preparation would also affect these processes. Our results in Fig. 3 for polycrystalline surfaces that have had no surface preparation, however, show virtually no variation despite a wide range in the target atomic number and density. Similar results were reported by Steiger et al..$^{10}$ for the formation of $n=2$ excited state Ps, which depends on the same nonthermal positron formation mechanism (the work functions for Ps excited states are positive). On the other hand, the velocity spectroscopy results of Howell et al. ${ }^{5}$ using UHV-prepared and cleaned singlecrystal surfaces show a clear trend with target atomic number that is consistent with backscattering of positrons.

The energy spectrum of the fast Ps has been observed ${ }^{5}$ to peak around $20 \mathrm{eV}$. This peak energy is also expected from the energy dependence of typical electron-capture cross sections. "The decrease in these cross sections at higher energies sets an effective upper limit on the fast Ps energy of roughly $100 \mathrm{eV}$. Combined with our estimate of a lower energy limit of about $6.5 \mathrm{eV}$, the deduced range in $P_{a}$ is thus constrained to $0.25<P_{a}<1$. The downward trend in $\lambda_{f}$ observed in Fig. 2 indicates that $P_{a}$ depends on the velocity of $P s$ and that fast Ps is formed over a range of energies.

Large values of $P_{a}$ caused by dissociation, or impact ionization, at the cavity surfaces are consistent with studies of the reflection of Ps from surfaces. Weber et al. ${ }^{12}$ found that Ps is reflected from a LiF crystal with high probability only for $E_{\mathrm{Ps}}<10 \mathrm{eV}$. As the Ps energy increased, reflectivity decreased presumably due to impact ionization. The result of impacl ionization is a dissociated positron that may annihilate directly or capture another electron from the surface. In the latter case there would be a $25 \%$ chance that it would form the short-lived parapositronium (singlet) state and thus $P_{a}$ would be at least 0.25 . Such a high value of $P_{a}$ for fast Ps is in sharp contrast with the results of experiments in which Ps is predominantly formed at energies below the dissociation threshold, where $P_{a}$ is almost immeasurably small, i.e., of order $10^{-5}$ (see Ref. 2). These results are typical of collisions of Ps with powder grains and gas molecules. Even the spin-exchange quenching of $o$-Ps due to collisions with adsorbed gases with unpaired electron spins can only result ${ }^{13}$ in $P_{a} \leqslant 10^{-2}$. While chemical binding of Ps to the surface can produce high values of $P_{a},{ }^{13}$ this process is unlikely at the high Ps kinetic energies present in this experiment.

To further test the hypothesis that fast Ps is collisionally quenching at the cavity surfaces with large $P_{a}$, we have studied the short-time behavior in a second positron beam which has much faster $(0.5 \mathrm{~ns})$ lime resolution (designed for depth-profiled positron lifetime spectroscopy ${ }^{7}$ ). The annihilation lifetime spectrum of a smaller, parallel-plate cavity (mean free path $=1 \mathrm{~cm}$ ) with a stainless-steel formation surface is shown in Fig. 4. The time delay between Ps formation and the first collision with a wall on the other side of the cavity is clearly evident as a mound or shoulder extending out to approximately $13 \mathrm{~ns}$. Beyond this shoulder the spectrum can be adequately fitted to two exponential components for which the short lifetime $\left(1 / \lambda_{f}\right)$ is $8-9$ ns, as expected

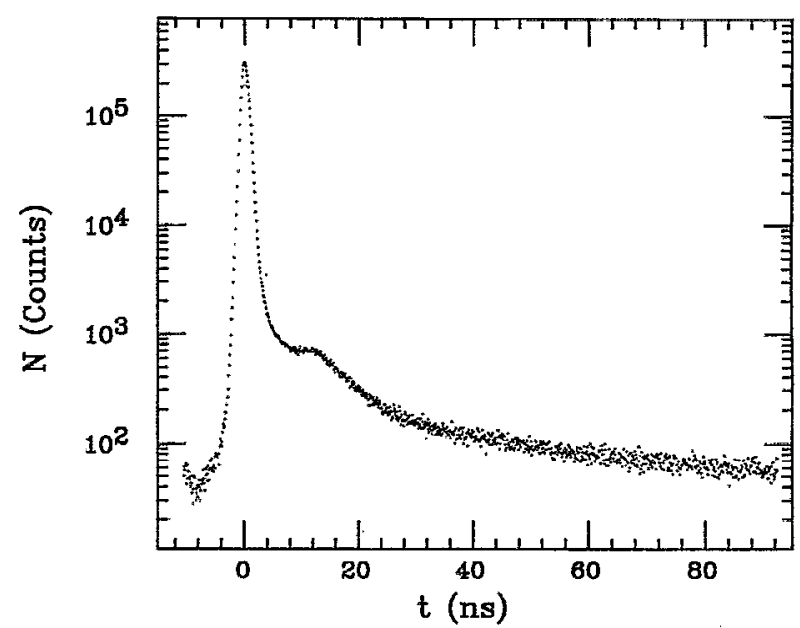

FIG. 4. The annihilation lifetime spectrum acquired in a separate positron beam designed for higher time resolution. Positrons strike a stainless-steel target at $1.2 \mathrm{keV}$ beam energy and any Ps formed is confined in a cavity with a mean free path of $1 \mathrm{~cm}$.

from Eq. (1) using $4 V / S=1 \mathrm{~cm}$. Furthermore, since the maximum distance from the Ps formation surface to the first collision is about $1.5 \mathrm{~cm}$, or roughly 1.5 mean collision lengths, it is reasonable that the shoulder terminus would occur near $t=13 \mathrm{~ns}$, or $1.5 \times$ the mean collision time. The detailed shape of the spectrum is indeed complicated (and depth-profiled lifetime spectroscopy will require further exploration ${ }^{7}$ ), but the general trends clearly support the model of fast Ps rapidly quenching at the cavity surfaces after a delay for time of flight.

More information on the Ps formation mechanism on metal surfaces can be found in Fig. 5, where we show the ratio of the intensity of the fast component (fit beginning at $t_{0}=42 \mathrm{~ns}$ ) to that of the $140 \mathrm{~ns}$ component. Despite the decrease in $I_{f}$ and $I_{T}$ by an order of magnitude with increasing positron implantation energy (Fig. 3), the ratio $I_{f} / I_{T}$ is

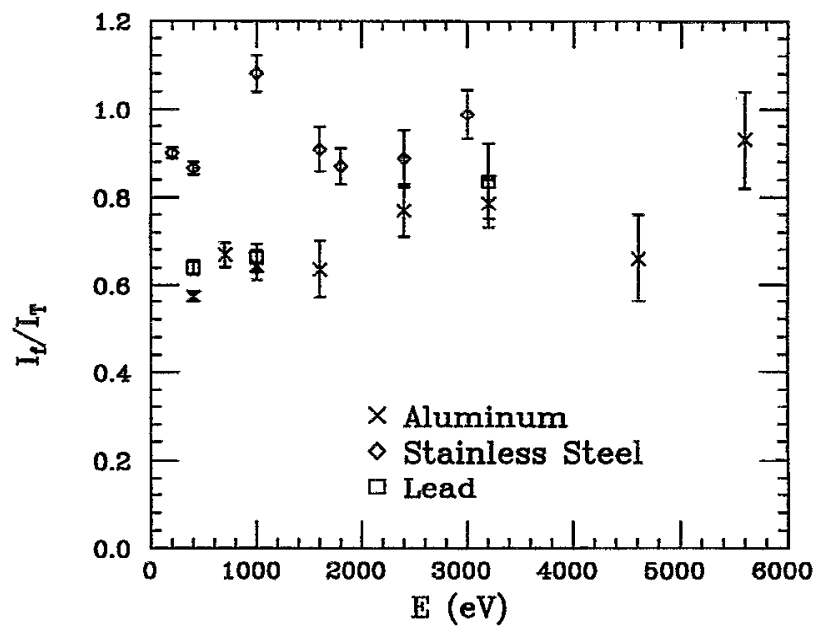

FIG. 5. Ratio of intensity of the short-lived Ps component, $I_{f}$, to the intensity of the long-lived $o$-Ps component, $I_{T}$, as a function of the positron beam energy $E$. 
quite independent of beam energy and only marginally dependent on the target material. This energy independence strongly suggests that these two components are both the result of Ps formation from nonthermalized positrons. On these machined metal surfaces, this mechanism is likely because thermalized positrons should be completely trapped in defects. Only energetic positrons can escape defect trapping and "diffuse" to the surface. It is not contradictory, however, to have a long-lived component produced by nonthermal positrons. The energy of emitted Ps formed from the capture of electrons well below the Fermi level should and does extend down to zero energy. ${ }^{9}$ The lower limit of about $6.5 \mathrm{eV}$ for the 17 ns component is simply indicative of a threshold energy for dissociation (presumably about $6.8 \mathrm{eV}$, the Ps binding energy), above which $P_{a}$ is near unity. For Ps with energies below the dissociation threshold, $P_{a}$ is negligibly small and the lifetime is close to $140 \mathrm{~ns}$. The constancy of $I_{f} / I_{T}$ in Fig. 5, and thus the ratio of fast to slow Ps, implies that the shape of the nonthermal positron energy spectrum at the surface is independent of the beam energy (at least for beam energies from 0.2 to $5 \mathrm{keV}$ ). In other words, higher implantation energy produces fewer nonthermal positrons, but with a similar energy distribution.

The results shown in Figs. 3 and 5 are quantitatively consistent with a model in which Ps is formed from a positron that has backscattered out of the incident beam. Studies of positron ${ }^{14}$ and electron ${ }^{15}$ backscattering show several general trends for the incident beam energies used in this experiment. First, the shape of the hackscattered spectrum is independent of beam energy $E$, and is nominally flat at backscattered energies less than $100 \mathrm{eV}$ where Ps formation can occur. This accounts for the constant value of $I_{f} / I_{T}$ observed in Fig. 5. Second, the backscattering coefficient (or fraction of incident positrons that backscatter) is largely independent of $E$. Thus the relative number of backscattered positrons at energies below $100 \mathrm{eV}$, and hence the relative number of fast Ps formed, is proportional to $E^{-1}$, in good agreement with the results shown in Fig. 3 and Monte Carlo calculations. ${ }^{16}$ On the other hand, models of Ps formation from the backdiffusion of thermalized positrons predict a beam energy dependence at least as strong as $E^{-1.6} .1$ We conclude that Ps in the fast component is formed from backscattered positrons.

We have attempted to fit the lifetime spectrum to a continuum distribution in $\lambda_{f}$, with a discrete peak at $\lambda_{T}$. If the distribution $n(\lambda)$ is known, then the energy distribution of fast Ps can be deduced (using reasonable assumptions for the energy dependence of $P_{a}$ ). We have not obtained acceptable fitting results (with real or simulated lifetime spectra) using the fitting program CONTIN. ${ }^{17}$ We have, instead, fitted two exponential components to simulated lifetime spectra that were generated using a variety of continuous distributions in $n(\lambda)$. The results for $\lambda_{f}$ are then compared with similar fitting of actual spectra (see Fig. 2). As expected, this approach does not determine a unique distribution function for $n(\lambda)$ as a variety of spectral shapes produces acceptable fits. One very clear result is that a broad distribution of Ps energies up to $\Rightarrow 70 \mathrm{eV}$ is required in all cases.

One feature seen in Fig. 3 that is relevant to precision Ps decay rate experiments is that fumed $\mathrm{MgO}$ powder is a poor choice for the formation surface because the fast Ps component is $3 \times$ larger than that for metals. There are two possible reasons for the large values of $F_{f}$ for $\mathrm{MgO}$. One is that the band gap in $\mathrm{MgO}$ prevents positrons from ever thermalizing ${ }^{18}$ in the bulk, and hence there are naturally more nonthermals $i^{z}$ available to produce fast Ps. A second reason is that fumed $\mathrm{MgO}$ consists of randomly oriented crystallites so that positrons are incident at all angles with respect to the crystallite surface. Backscattering is enhanced at non-normal incidence $^{14}$ and averaging over a random distribution of - angles might easily account for the observed enhancement in $\mathrm{MgO}$. We also note that there is no indication of Ps trapping in the $\mathrm{MgO}$ powder pores ${ }^{2}$ since the $\mathrm{MgO}$ data of Fig. 3 parallel the metal results so closely. If present, pore trapping should increase as the beam energy increases so that positrons can penetrate deeper below the surface to reach the pores. In powder decay rate measurements ${ }^{19}$ using beta decay radioactive sources (with very deep implantation depths), pore trapping may still be important. It is not important in cavity decay rate experiments with slow positron beams.

\section{CONCLUSION}

We conclude that collisional dissociation of Ps atoms with energies of order 10-100 eV produces lifetime components in the time spectrum that decay faster than Ps that is formed at a few electron volts. The fast component decay rates do not appear to be affected by the positron beam implantation energy, but the intensities decrease roughly as $E^{-1}$, consistent with Ps formation from backscattered positrons. The fast component decay rates are observed to scale inversely with the cavity mean free path as expected from collisional dissociation. Backscattered positrons can also form low-energy Ps (which has negligible collisional dissociation) with a relative intensity that is approximately equal to the fast Ps component.

In order to minimize the effect of these fast decaying components, future precision Ps decay rate experiments in evacuated cavities should (1) utilize the highest beam energy consistent with sufficient $\mathrm{Ps}$ formation in the long-lived 140 ns component, (2) switch from MgO to metal surfaces where normal incidence of the beam to the surface can be obtained, and (3) utilize small cavities with short mean free paths in order to quench the fast Ps as quickly as possible. Lowenergy depth-profiling experiments that have recently implemented positron annihilation lifetime spectroscopy ${ }^{6,7}$ will have to account for the effect of dissociating Ps (and its energy dependence) on the lifetime spectrum. Depth profiling is accomplished by varying the beam implantation energy. In these experiments the emphasis is on measuring the lifetimes and intensities of positron and Ps components in the first $\sim 10$ ns of the spectrum (lifetimes $<3 \mathrm{~ns}$ ). Therefore, one can minimize the systematic effects of fast Ps collisions by maximizing the distance (and hence the time) to the first wall collision (i.e., utilize large cavitics) and fitting the spectrum as close to the prompt peak as possible. This approach has recently been used in our laboratory with success. ${ }^{7}$ It is found that the fast Ps component is well approximated by a 
flat background that extends out to the shoulder terminus at $t \approx 13 \mathrm{~ns}$ (see Fig. 4).

\section{ACKNOWLEDGMENTS}

We thank all members of the Michigan Positron Group (past and present) for many hours of discussion and research concerning the origin of the fast Ps component. In particular, we àcknowledge the undergraduate thesis research ${ }^{20}$ of Aaron Noble that set the stage for this experiment. This work is supported by the National Science Foundation Grants No. PHY-9119899 and No. PHY-9205195 and the University of Michigan.

${ }^{1}$ For an excellent review, see P. J. Schultz and K. G. Lynn, Rev. Mod. Phys. 60, 701 (1988).

${ }^{2}$ J. S. Nico, D. W. Gidley, A. Rich, and P. W. Zitzewitz, Phys. Rev. Lett. 65, 1344 (1990); J. S. Nico, Ph.D. thesis, University of Michigan, 1991.

${ }^{3}$ M. S. Fee, A. P. Mills, Jr., S. Chu, E. D. Shaw, K. Danzman, R. J. Chichester, and D. M. Zuckerman, Phys. Rev. Lett. 70. 1397 (1993); D. Hagena, R. Ley, D. Weil, G. Werth, W. Arnold, and H. Schneider, ibid. 71, 2887 (1993).

${ }^{4}$ H. Huomo, A. Vehanen, M. D. Bentzon, and P. Hautojärvi, Phys. Rev. B 35, 8252 (1987).

${ }^{5}$ R. H. Howell, I. J. Rosenberg, and M. J. Fluss, Phys. Rev. B 34, 3069 (1986).

${ }^{6}$ Y. C. Jean, G. H. Dai, H. Shi, R. Suzuki, and Y. Kobayashi, in Slow
Positron Beam Techniques for Solids and Surfaces, edited by Eric Ottewitte and Alex Weiss (AIP, New York, 1994), p. 129.

${ }^{7}$ L. Xie, G. Demaggio, J. DeVries, W. Frieze, D. Gidley, H. Hristov, and A. Yee, Phys. Rev. Lett. (to be published).

${ }^{8}$ Y. C. Jean, Microchem. J. 42, 72 (1990).

${ }^{9} \mathrm{See}$, for example, R. H. Howell, I. J. Rosenberg, M. J. Fluss, R. E. Goldberg, and R. B. Laughlin, Phys. Rev. B 35, 5303 (1987).

${ }^{10}$ T. D. Steiger and R. S. Conti. Phys. Rev. A 45, 2744 (1992).

${ }^{11}$ See, for example, W. Raith, in Atomic Physics with Positrons, edited by J. W. Humberston and E. A. G. Armour (Plenum, New York, 1987), p. 1, and references therein.

${ }^{12}$ M. H. Weber, S. Tang, S. Berko, B. L. Brown, K. F. Canter, K. G. Lynn, A. P. Mills, Jr., L. O. Roellig, and A. J. Viescas, Phys. Rev. Lett. 61, 2542 (1988).

${ }^{13}$ R. L. Klobucher and P. J. Karol, J. Phys. Chem. 84, 489 (1980).

${ }^{14}$ P. G. Coleman, L. Albrecht, K. O. Jensen, and A. B. Walker, J. Phys. Condens. Matter 4, 10311 (1992), and references therein; G. R. Massoumi, W. N. Lennard, P. J. Schultz, A. B. Walker, and K. O. Jensen, Phys. Rev. B 47, 11007 (1993).

${ }^{15}$ E. J. Sternglass, Phys. Kev. 95, 345 (1954).

${ }^{16}$ K. A. Ritley, K. G. Lynn, V. J. Ghosh, D. O. Welch, and M. McKeown, J. Appl. Phys. 74, 3479 (1993).

${ }^{17}$ R. B. Gregory, Nucl. Instrum. Methods A 302, 496 (1991), and references therein.

${ }^{18}$ P. Sferlazzo, S. Berko, and K. F. Canter, Phys. Rev. Lett. 35, 5315 (1987).

${ }^{19}$ D. W. Gidley, K. A. Marko, and A. Rich, Phys. Rev. Lett. 36, 395 (1976); D. W. Gidley, Ph.D. thesis, University of Michigan, 1979.

${ }^{20}$ A. A. Noble, Undergraduate Honors Thesis in Physics, The University of Michigan, 1992. 